\title{
Comparison of Various Methods for Calculating the AC Resistance of Inductors
}

\author{
Alberto Reatti and Marian K. Kazimierczuk, Senior Member, IEEE
}

\begin{abstract}
The paper reviews several expressions for high-frequency winding resistance of inductors proposed by several authors and compares the theoretical predictions calculated from these expressions with experimental results. It identifies the expressions that yield the most accurate prediction of the winding high-frequency resistance. The comparison shows that the method proposed by Dowell accurately predicts the ac resistance if the winding contains less than three layers. The methods proposed by several other authors accurately predict the high-frequency resistance only in certain frequency ranges. In addition, these expressions yield inaccurate results for the inductor quality factor. One expression, however, accurately predicts both the high-frequency winding resistance and the quality factor of inductors over a wide frequency range from the dc to the first resonant frequency. The paper concludes with a simple and accurate circuit model describing the frequency behavior of inductors.
\end{abstract}

Index Terms-High-frequency resistance, magnetics, power inductors.

\section{INTRODUCTION}

$\mathbf{T}$ HE OVERALL efficiency of a power converter highly depends on the efficiency of power inductors used in assembling the power converter circuit. Therefore, the control of power losses in these components is important in designing high-efficiency power converters. One of the main problems related to the design of power inductors is the calculation of the winding ac resistance $R_{\text {ac. }}$. Many efforts [1]-[15] have been made to derive expressions allowing for an accurate representation of frequency behavior of $R_{\mathrm{ac}}$. The objectives of this paper are: 1) to compare the expressions for the inductor winding ac resistance $R_{\mathrm{ac}}$ proposed by several authors; 2) to confront the theoretical predictions with experimental results; and 3) to identify the most accurate expression describing $R_{\mathrm{ac}}$. It is shown that the error of calculating the quality factor $Q$ of inductors is very sensitive to the error of calculating the ac resistance $R_{\mathrm{ac}}$. Therefore, an accurate expression for $R_{\mathrm{ac}}$ is required to accurately predict $Q$.

The significance of the paper is that the comparison of several methods allows for the identification of the method resulting in the most accurate prediction of the frequency behavior of an inductor winding ac resistance. Both the expression for $R_{\mathrm{ac}}$ and the inductor model can be used to design inductors for highfrequency operation with reduced winding losses.

Manuscript received December 13, 1999; revised January 7, 2002.

A. Reatti is with the Department of Electronics and Telecommunications, University of Florence, 50139 Florence, Italy (e-mail: reatti@ det.unifi.it).

M. K. Kazimierczuk is with the Department of Electrical Engineering, Wright

State University, Dayton, OH 45435 USA (e-mail: mkazim@cs.wright.edu).

Publisher Item Identifier S 0018-9464(02)03636-1.

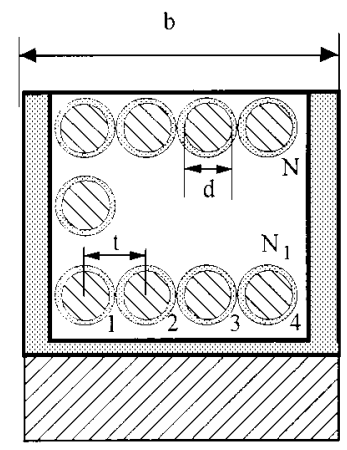

(d)

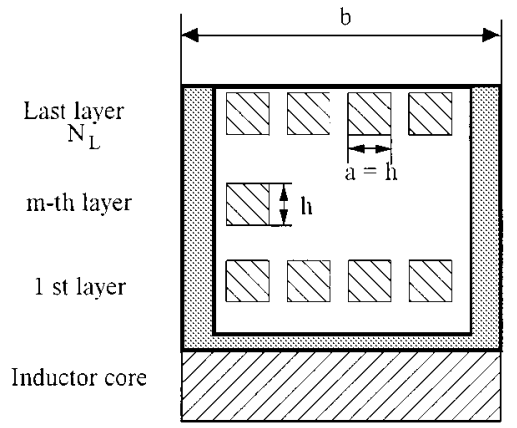

(b)
Fig. 1. Cross section of an inductor winding. (a) With round wire. (b) With square wire.

\section{EXPRESSIONS FOR THE WINDING AC RESISTANCE}

\section{A. Dowell Method}

The expression for the winding ac resistance given in [1] is determined by exploiting the one-dimensional solution of the field in the winding space. Fig. 1 shows cross sections of two windings: one with a round wire and the other with a square wire. Most of the symbols used in the following equations are explained in Fig. 1.

1) The magnetic field distribution is solved for a "winding portion." As shown in Fig. 2, a winding portion is defined in [1] as a part of the transformer winding, which extends in either direction along the axis of the winding height from a position of zero field intensity to the first positive or negative peak of the magnetic field intensity.

2) The magnetic flux in the transformer winding space is parallel to the core leg.

3) The conductor layers are modeled as continuous conductor foils, which fill the full core-window breath. The expressions for $R_{\mathrm{ac}}$ of round conduction wires is derived by first considering an equivalent square cross-sectional area conductor, and then finding an equivalent foil conductor with the same dc resistance of the round and square wires. For this purpose, the porosity factor is introduced as

$$
\eta=\frac{a N_{T L}}{b}
$$

where $a=h$ is the width of the square conductor, $b$ is the width of the inductor bobbin, and $N_{\mathrm{TL}}$ is the number of turns in one layer of the winding. 


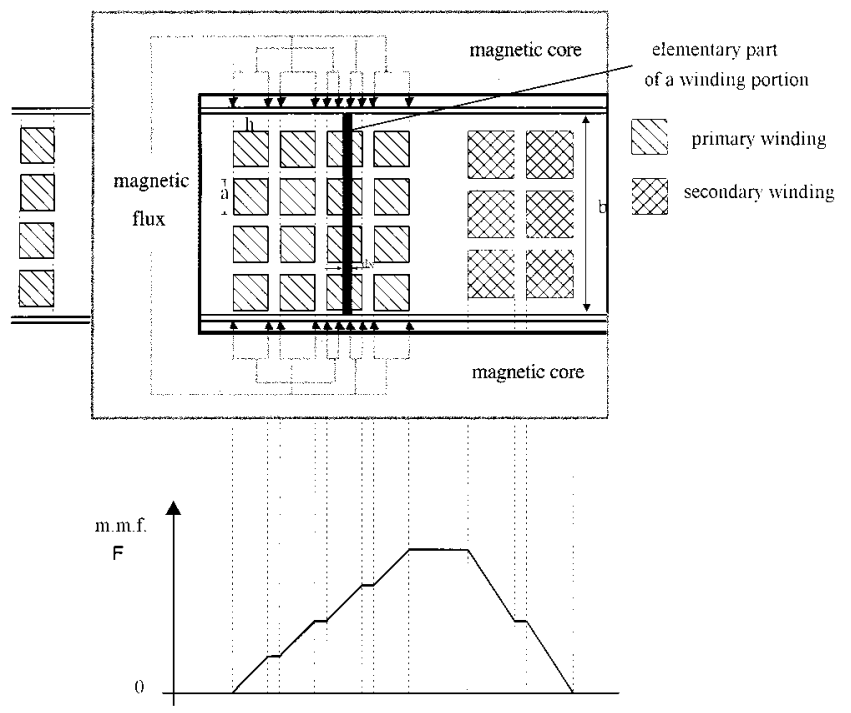

Fig. 2. Winding portion.

4) The curvature of the conductors is neglected when calculating the radial field distribution across the winding layer.

5) Capacitive effect in the winding is ignored.

6) The magnetic field intensity of any winding layer is negligible outside that layer.

7) The expression for the winding ac resistance of a solid square cross-sectional area wire inductor given in [1] is

$$
\begin{aligned}
R_{\mathrm{ac}}=R_{\mathrm{dc}} A\left[\frac{e^{2 A}-e^{-2 A}+2 \sin 2 A}{e^{2 A}+e^{-2 A}-2 \cos 2 A}\right. \\
\left.+\frac{2}{3}\left(m^{2}-1\right) \frac{e^{2 A}-e^{-2 A}-2 \sin 2 A}{e^{2 A}+e^{-2 A}+2 \cos 2 A}\right]
\end{aligned}
$$

where

$R_{\mathrm{dc}}=r_{L} N l_{T}=\mathrm{dc}$ winding resistance;

$r_{L} \quad=$ winding resistance per unit length $\left(r_{L}=4 \rho / \pi d^{2}\right.$ for a solid round conductor wire with a copper diameter $d$ );

$\rho \quad=1 / \sigma=17.2410-6 \Omega \mathrm{mm}=$ copper resistivity at $T=20^{\circ} \mathrm{C}$, where $\sigma=1 / \rho$ is the wire conductivity;

$l_{T} \quad=$ average length of one winding turn;

$N \quad=$ number of turns in the winding;

$m \quad=$ number of layers in one winding portion;

$A \quad=h / \delta$ for a square cross-sectional area conductor

$$
A=\frac{h}{\delta} \sqrt{h}=\frac{d}{\delta} \sqrt{\frac{4}{\pi} \eta}=\left(\frac{4}{\pi}\right)^{3 / 4} \frac{d^{3 / 2}}{\delta t^{1 / 2}}
$$

for round cross-sectional area conductor [2] in which

$h=$ copper side of a square cross conductor section;

$d \quad=$ copper diameter of a round conductor wire;

$t=$ distance between the centers of two adjacent conductors;

$\eta \quad=d / t$ is the porosity factor for a solid round wire;

$\delta \quad=1 / \sqrt{\pi \mu_{o} \mu_{r} \sigma f}=$ skin penetration depth;

$\mu_{o}=4 \pi \times 10^{-7} \mathrm{H} / \mathrm{mm}=$ free air magnetic permeability;

$\mu_{r}=$ relative permeability $\left(\mu_{r}=1\right.$ for a copper conductor).
The assumption that the magnetic flux is parallel to the winding layers is not well satisfied for low values of $\eta(\eta \leq 0.7)$. Therefore, the application of (2) to solid round wires results in a good accuracy of $R_{\mathrm{ac}}$ only for windings with high values of the porosity factor $\eta(0.7 \leq \eta \leq 1)$. The major limitation of (2) is that it cannot be used to represent the ac resistances of bunched and Litz wires [3]. The results of (2) have been used in [4] to derive the ac resistance of winding conducting nonsinusoidal currents. Moreover, the equations given in [1] apply only to two layers of conductors and exactly at the center of the layer. This means that (2) is strictly correct when solved for the winding portion shown in Fig. 2 contained between the point where the m.m.f. $\Im=0$ and the point where the m.m.f. $\Im$ returns to zero after reaching its maximum value. Therefore, this approach is not suitable for analyzing transformers with three or more windings.

\section{B. Perry, Bennet, and Larson Method}

Whereas the magnetic field equations are solved for a winding portion in [1], an analysis based on general field solutions for the distribution of current density in a single layer of an infinitely long, cylindrical current sheet is followed in [5] and [6]. Consequently, although the author of [5] is interested only in multilayer solenoids (air-core inductors), his method is applicable to multiwinding transformers as well. The ac resistance of the $m$ th layers of the winding coil is

$$
\begin{aligned}
R_{\mathrm{ac}-m}=R_{\mathrm{dc}} \frac{\xi}{2}\left[\frac{\sinh \xi}{\cosh \xi}-\sin \xi\right. & \cos \xi \\
+ & \left.\frac{2}{3}\left(m^{2}-1\right) \frac{\sinh \xi-\sin \xi}{\cosh \xi+\cos \xi}\right]
\end{aligned}
$$

where $\xi=d \sqrt{\pi} / 2 \delta$ is the thickness of the conductor foil normalized with respect to the skin depth $\delta$. Using (4), the derivation of $R_{\mathrm{ac}}$ for multilayer inductors can easily be performed. It is shown in [7] that this approach can be used to determine the losses in a multiwinding transformer conducting nonsinusoidal currents.

In [5] and [6], a procedure is given to derive an expression for $R_{\mathrm{ac}}$ in the cylindrical coordinates by using the Bessel functions. This allows for the curvature of a wire wound on a bobbin to be taken into account. However, the derivation of $R_{\mathrm{ac}}$ is cumbersome and refers to infinitely long conductor foils. Therefore, the application of this procedure to round wire winding requires approximations resulting in an inaccurate prediction of $R_{\mathrm{ac}}$. Several details on the methods proposed in [1]-[7] are given in [8].

\section{Ferreira-1 Method}

The expression for $R_{\mathrm{ac}}$ given in [5] has been rearranged in [9] to be extended also to square cross-sectional area wires. For this purpose, a single layer skin depth $\delta=1 / \sqrt{\pi \mu_{O} \mu_{r} \sigma f \eta}$ has been considered and the expression of the $m$ th layer ac resistance has been derived in the cylindrical coordinates. The final result is

$$
\begin{aligned}
R_{\mathrm{ac}-m}=R_{\mathrm{dc}} \frac{\xi(\eta)}{2} & {\left[\frac{\sinh \xi(\eta)+\sin \xi(\eta)}{\cosh \xi(\eta)-\cos \xi(\eta)}\right.} \\
& \left.+\eta^{2}(2 m-1) \frac{\sinh \xi(\eta)-\sin \xi(\eta)}{\cosh \xi(\eta)+\cos \xi(\eta)}\right]
\end{aligned}
$$


where $\xi(\eta)=d \sqrt{\pi} /(2 \delta)$. The squared porosity factor $\eta^{2}$ multiplying the second term of (5) represents the ratio of the wire effective cross-sectional area carrying the current to the overall conductor cross-sectional area and shows that the proximity effect highly contributes to the increase of ac resistance with frequency. The main limitation of (5) is that this equation is derived for foil conductors and square cross-sectional area wires and, therefore, approximations are introduced when it is applied to round wires.

\section{Ferreira-2 Method}

The winding ac resistance of the $m$ th layer derived in the cylindrical coordinates by solving the field equations for round wires is [10]

$$
\begin{aligned}
& R_{\mathrm{ac}-m}=R_{\mathrm{dc}} \frac{\gamma}{2}\left[\frac{b e r \gamma b e i^{\prime} \gamma-b e r \gamma b e i^{\prime} \gamma}{b e r^{2} \gamma+b e i^{2} \gamma}\right. \\
& \left.+2 \pi(2 m-1) \frac{b e r_{2} \gamma b e i^{\prime} \gamma-b e r_{2} \gamma b e i^{\prime} \gamma}{b e r^{2} \gamma+b e i^{2} \gamma}\right] .
\end{aligned}
$$

This expression should be more accurate than (4) and (5) when used for round wires. However, it does not take into account the contribution of $\eta^{2}$, where $\eta$ is the ratio of the wire cross area carrying the current to the overall conductor cross-sectional area and show that the proximity effect highly contributes to increase the ac resistance with frequency. As a consequence, (6) results in an inaccurate prediction of $R_{\mathrm{ac}}$.

\section{E. Reatti and Kazimierczuk Method}

The previous methods followed a multidimensional approach by assuming a uniform distribution of the magnetic field across the conductor cross-sectional area. This assumption is removed in [11] and an expression that gives an accurate prediction of the winding ac resistances is of the form

$$
\begin{aligned}
& R_{\mathrm{ac}-m}=R_{\mathrm{dc}} \frac{\gamma}{2}\left\{\frac{\text { ber } \gamma \text { bei } \gamma-\text { bei } \gamma \text { ber' } \gamma}{\text { ber }^{2} \gamma+b e i^{2} \gamma}\right. \\
& \left.-2 \pi \eta^{2}\left[\frac{4\left(N_{l}^{2}-1\right)}{3}+1\right] \frac{\text { ber }_{2} \gamma b e i \gamma-b e i_{2} \gamma b e i \gamma}{b e r^{2} \gamma+b e i^{2} \gamma}\right\} .
\end{aligned}
$$

\section{COMPARISON OF THE EXPRESSIONS AND EXPERIMENTAL RESULTS}

To compare the theoretical results calculated by using (2) and (4)-(7) and the experimental results, the equivalent circuit of inductors shown in Fig. 3 was used [11]-[14]. In Fig. 3(a), $L$ is the nominal inductance, $R_{c}$ is the magnetic core resistance, and $C$ is the inductor self-capacitance. Both resistances $R_{\mathrm{ac}}$ and $R_{c}$ increase with frequency. If the core resistance $R_{c}$ is much lower than the winding ac resistance $R_{\text {ac }}$, the inductor model simplifies to the form depicted in Fig. 3(b). Most LCR meters measure an equivalent series reactance $X_{s}$ and an equivalent series resistance of two-terminal devices as shown in Fig. 3(c). The impedance of the equivalent circuit depicted in Fig. 3(c)

$$
Z_{S}=R_{S}+j X_{S}=\frac{R_{\mathrm{ac}}+j \omega L\left(1-\omega^{2} L C-C R_{\mathrm{ac}}^{2} / L\right)}{\left(1-\omega^{2} L C\right)^{2}+\left(\omega L R_{\mathrm{ac}}\right)^{2}} .
$$

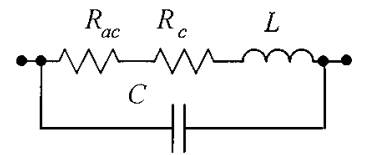

(a)

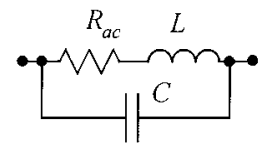

(b)

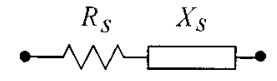

(c)

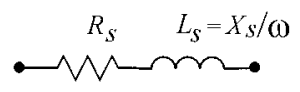

(d)
Fig. 3. Equivalent circuits of inductors. (a) Lumped parameter equivalent circuit. (b) Simplified lumped parameter equivalent circuit for $R_{c} \ll R_{\mathrm{ac}}$. (c) Series equivalent circuit. (d) Equivalent circuit assumed by many LCR meters.

For frequencies $f$ much lower than the first self-resonant frequency $f_{r}$, the equivalent series reactance $X_{s}$ has an inductive character and can be expressed as $X_{s}=\omega L_{s}$. Hence, the equivalent series inductance is $L_{s}=X_{s} / \omega$ as shown in Fig. 3(d). The quality factor of the inductor at a given frequency is defined as

$$
Q=\frac{\left|X_{S}\right|}{R_{S}}=\frac{\left|\omega L\left(1-\omega^{2} L C-C R_{\mathrm{ac}}^{2} / L\right)\right|}{R_{\mathrm{ac}}} .
$$

In order to separate the winding resistance $R_{\mathrm{ac}}$ from the core resistance $R_{c}$, an air-core (coreless) inductor and an inductor with an iron-powder core were chosen for experimental tests. For air-core inductors, the core resistance is zero. For inductors with iron-powder cores, the core resistance $R_{c}$ is much lower that the winding resistance $R_{\mathrm{ac}}$. The two inductors were assembled and tested with an HP4192A LF Impedance Analyzer equipped with an HP16047A test fixture to achieve a higher accuracy in minimizing residual parameters and contact resistances.

\section{A. Inductor no. 1}

A two-layer winding air-core inductor with $N=146\left(N_{l}=\right.$ 73) of an AWG\#25 wire, with the copper diameter $d=0.45 \mathrm{~mm}$ and the winding pitch $t=0.65 \mathrm{~mm}$ was wound on a plastic toroidal bobbin with an effective area $A_{e}=32 \mathrm{~mm}^{2}$, an effective magnetic length $l_{e}=50 \mathrm{~mm}$, an effective volume $V_{e}=$ $1600 \mathrm{~mm}^{3}$, and an average turn length $l_{T}=26.8 \mathrm{~mm}$. The core resistance $R_{c}$ was zero in this case. The inductance measured at low frequencies was $L=25 \mu \mathrm{H}$. At low frequencies, the inductance $L$ and the series inductance $L_{s}$ were equal. The measured first resonant frequency was $f_{r}=4.935 \mathrm{MHz}$.

\section{B. Inductor no. 2}

A three-layer winding inductor with $N=114\left(N_{l}=38\right)$ of an AWG\#28 wire with $d=0.32 \mathrm{~mm}$ and $t=0.393 \mathrm{~mm}$ was wound on a Micrometals E-25 iron-powder core. The relative permeability of this core with no air gap was $\mu_{r}=75$. A $9 \mathrm{~mm}$ air gap was introduced on the central leg to reduce the effective relative permeability to $\mu_{e}=6$. For powder core inductors, the core resistance $R_{c}$ is much lower than the winding resistance $R_{\text {ac }}$. The inductance measured at low frequencies was $L=166 \mu \mathrm{H}$. The measured first self-resonant frequency was $f_{r}=2.039 \mathrm{MHz}$. 

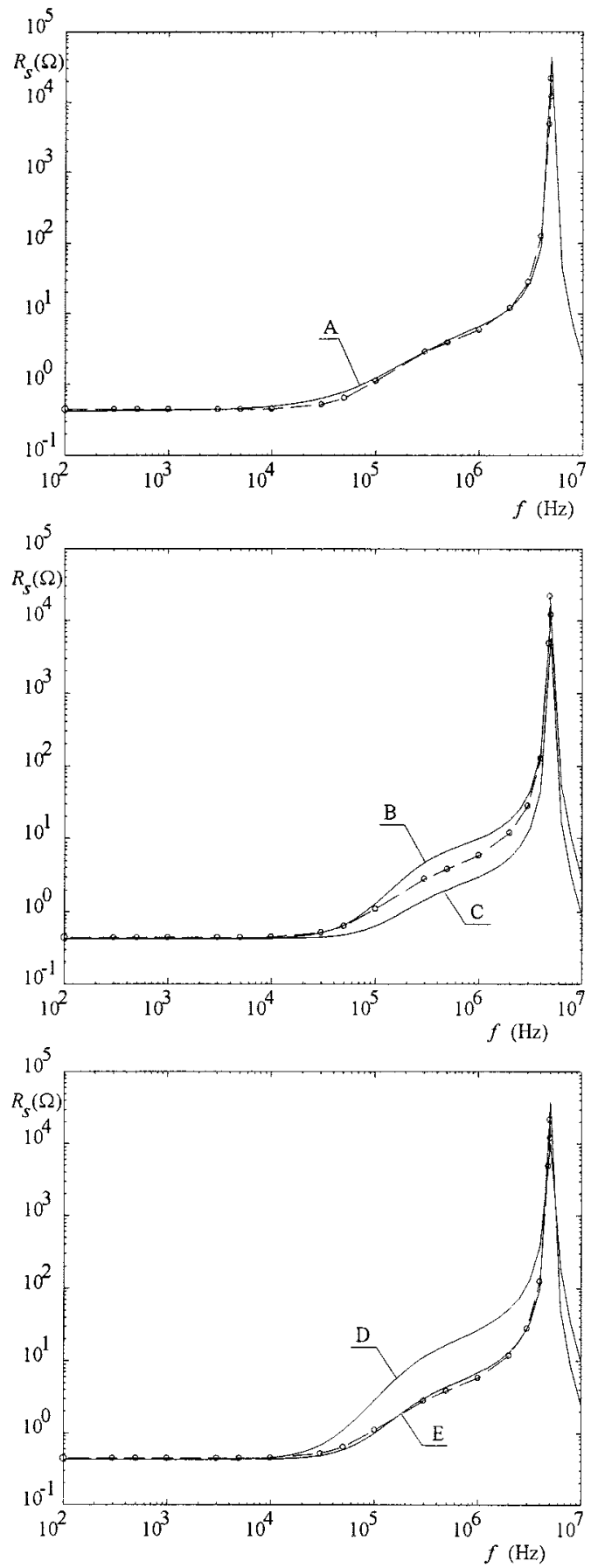

Fig. 4. Equivalent series resistance $R_{s}$ of inductor no. 1. A-Dowell; B-Perry; C-Ferreira 1; D-Ferreira 2; E-Reatti; O-O-Experimental.

\section{Measured and Calculated Results}

The plots of the calculated and measured equivalent series resistance $R_{s}$ of inductor no. 1 are shown in Fig. 4 . The expression for $R_{\text {ac }}$ given by (2) leads to the calculated $R_{s}$ that is in good agreement with the measured $R_{s}$. However, (4)-(6) yield an accurate prediction of $R_{s}$ only in the low-frequency range $(f<10-20 \mathrm{kHz})$. Equation (7) gives an accurate calculation of $R_{s}$ up to the inductor first self-resonant frequency.

The measured and calculated plots of the quality factor $Q$ of the inductor are depicted in Fig. 5. Using (2), good agreement of theoretical and experimental results is achieved for frequencies
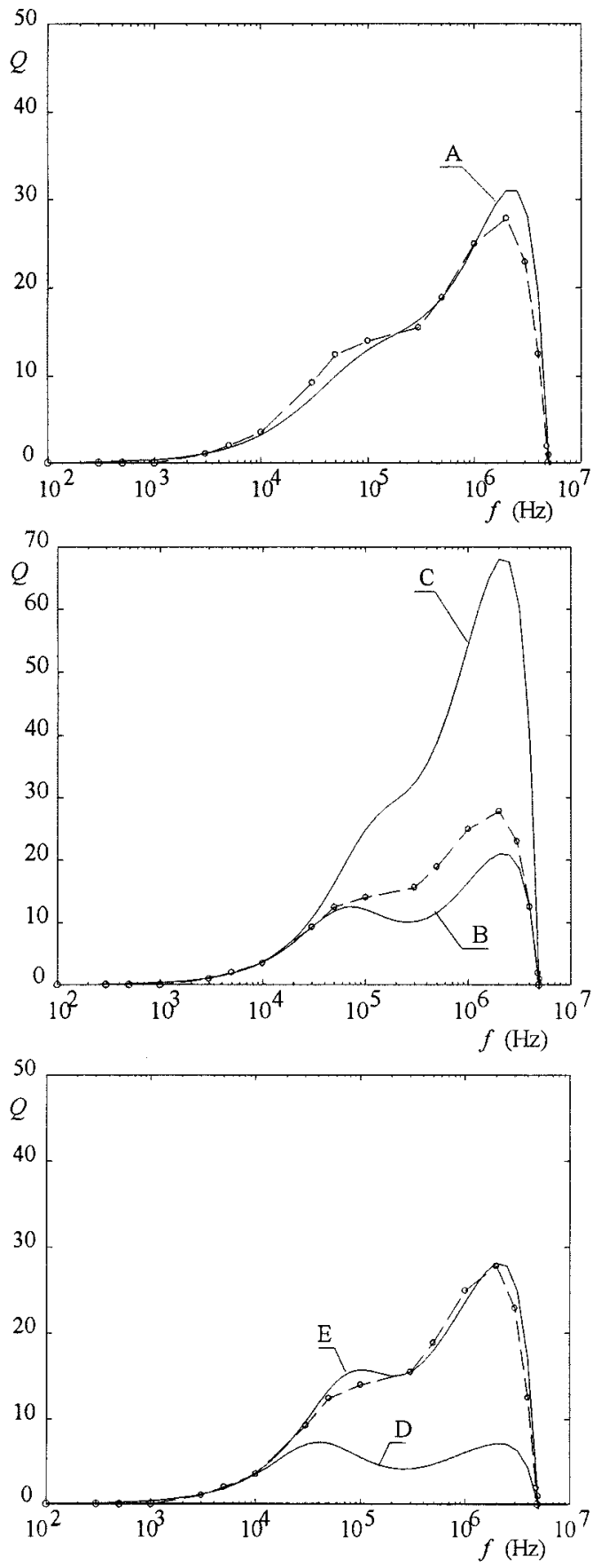

Fig. 5. Quality factor $Q$ of inductor no. 1. A-Dowell; B-Perry; C-Ferreira 1; D-Ferreira 2; E-Reatti; O-O-Experimental.

up to $5 \mathrm{kHz}$ and from 200 to $800 \mathrm{kHz}$. Plots B, C, and D show calculated values of $Q$ very much different from the measured values. Plot $\mathrm{E}$ is in good agreement with the experimental plot. A maximum $15 \%$ error occurs at $f \approx 70 \mathrm{kHz}$. The plots of the equivalent series resistance $R_{s}$ of the inductor no. 2 are shown in Fig. 6. Plot A is in good agreement with the measured plot only at low frequencies, e.g., $f \leq 2 \mathrm{kHz}$.

On the other hand, plot B is in good agreement with the measured plot up to the first resonant frequency. There are large errors for curves $\mathrm{C}$ and $\mathrm{D}$ for $f \geq 10 \mathrm{kHz}$, whereas plot $\mathrm{E}$ is in good agreement with the measured values over the entire frequency range. Fig. 7 shows the plots of the measured 

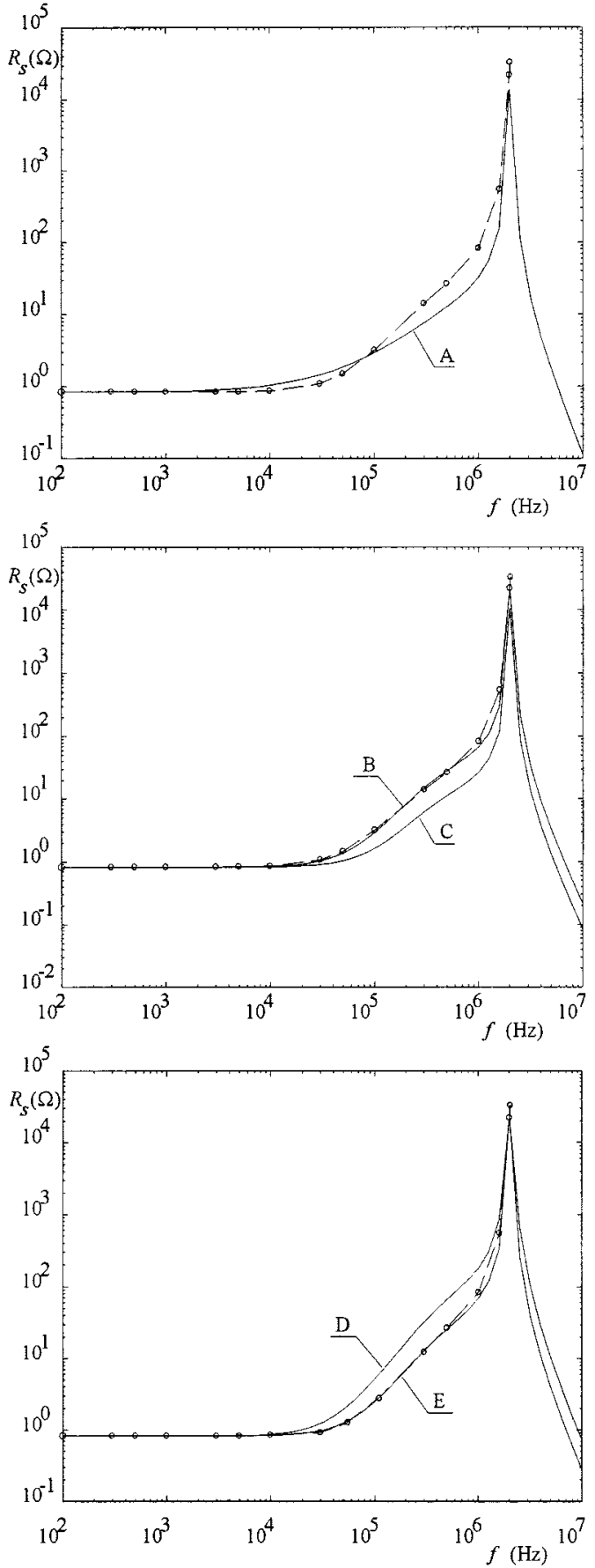

Fig. 6. Equivalent series resistance $R_{s}$ of inductor no. 2. A-Dowell; B-Perry; C-Ferreira 1; D-Ferreira 2; E-Reatti; O-O-Experimental.

and calculated values of quality factor $Q$ of the inductor no. 2. Plots A, C, and D show that (4), (5), and (6) yield values of $R_{\mathrm{ac}}$, resulting in large approximated calculated quality factors. Plots $\mathrm{B}$ and $\mathrm{E}$ are in good agreement with the measured plot.

The plots of $R_{\mathrm{s}}$ shown in Figs. 4 and 6 demonstrate that the expression (2) for $R_{\mathrm{ac}}$ results in an accurate prediction of an inductor equivalent series resistance $R_{s}$ for two-layer inductors, but it becomes less accurate for inductors with more than two layers. Similarly, expression (4) allows for calculated values of $R_{s}$ to be in agreement with measured values only in certain
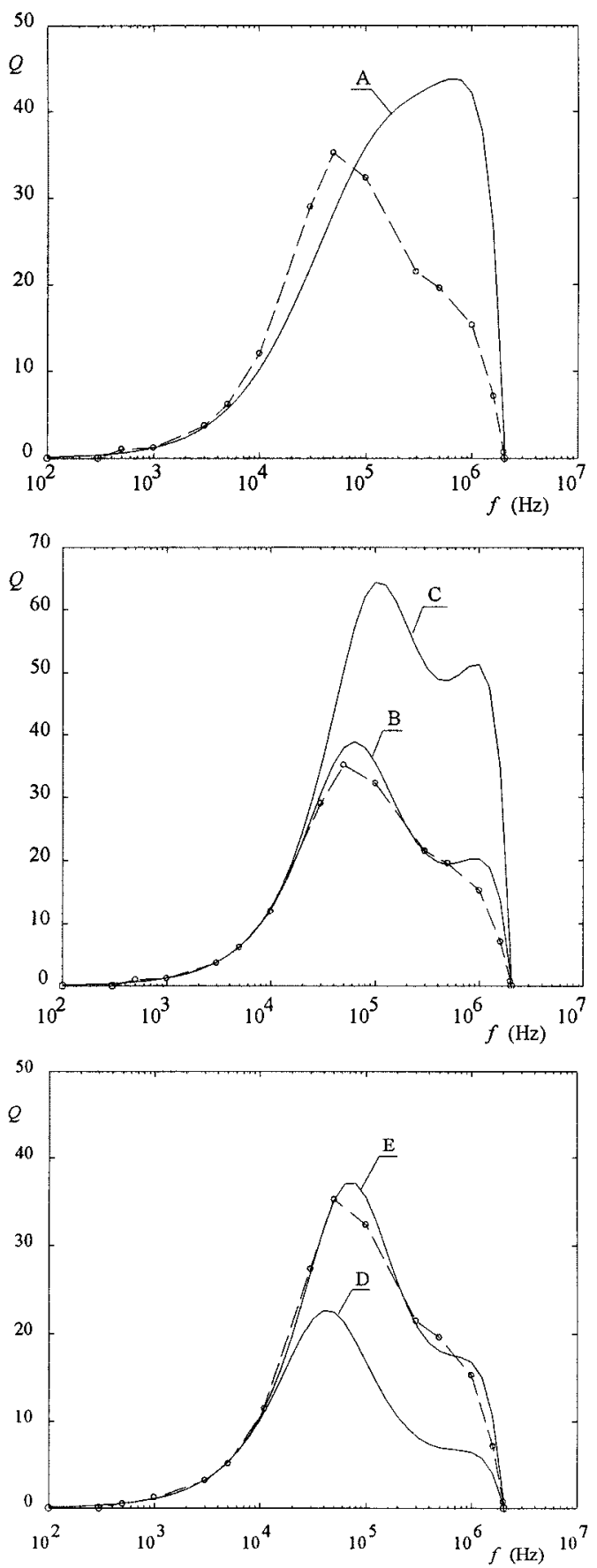

Fig. 7. Quality factor $Q$ of inductor no. 2. A-Dowell; B-Perry; C-Ferreira 1; D-Ferreira 2; E-Reatti; O-O-Experimental.

cases, e.g., inductor no. 2. Calculated values of $R_{\text {ac }}$ resulting from (5) and (6) yield values of $R_{s}$, which are accurate only in the low frequency range, e.g., $f \leq 10 \mathrm{kHz}$ for inductor no. 1 and $f \geq 20 \mathrm{kHz}$ for inductor no. 2. As shown in the Appendix, whenever the expression for $R_{\mathrm{ac}}$ results in a large error of $R_{s}$, the percent error of $Q$ is as high as $100 \%$. This explains the large differences between the measured and calculated values of $Q$, yielding plots B, C, and D of Fig. 5 and A, C, and D of Fig. 7.

Plots $\mathrm{E}$ of $R_{s}$ shown in Figs. 4 and 6 demonstrate that expression (6) for $R_{\mathrm{ac}}$ allows for an accurate prediction 
of an inductor equivalent series resistance independently from the inductor number of layers, inductor shape, etc. A very good accuracy of calculating the inductor resistance is demonstrated by the plots of $Q$ depicted in Figs. 5 and 7. As shown in the Appendix, the calculated values of $Q$ are in good agreement with measured values only if $R_{s}$ is predicted very accurately.

\section{CONCLUSION}

Several expressions for an inductor high-frequency winding resistance have been compared. Plots of $R_{\mathrm{ac}}$ calculated from these expressions have been also compared with experimental results. The inductors used for the experimental tests were assembled using a care such that the core resistance was negligible compared to the winding resistance.

It has been shown that most methods allow for an accurate prediction of the inductor winding ac resistance only in certain frequency ranges. Moreover, most of them yield accurate results only for certain winding compositions. Since large errors for the inductor equivalent series resistance result in large errors for the inductor quality factor, most of the compared methods fail in describing the frequency behavior of the quality factor. Only the expression (6) given in [11] allows for an accurate prediction of the ac resistance from dc to the inductor first resonant frequency independently from the inductor winding composition. Moreover, the small errors achieved for the calculated inductor equivalent series resistance allow for an accurate calculation of the quality factor over a wide frequency range. Expression (6) and the simple inductor model given in the paper can be used for designing inductor windings with reduced high-frequency resistances.

\section{APPENDIX \\ CALCUlation ERRORS OF THE INDUCTOR RESISTANCE AND QUALITY FACTOR}

The percentage error of calculating $R_{\mathrm{ac}}$ is

$$
\frac{\Delta R_{\mathrm{ac}}}{R_{\mathrm{ac}-m}} \%=\frac{R_{\mathrm{ac}-c}-R_{\mathrm{ac}-m}}{R_{\mathrm{ac}-m}} \times 100
$$

where $R_{\mathrm{ac}-c}$ and $R_{\mathrm{ac}-m}$ are the calculated and measured resistances. The percentage errors of calculating the inductor quality factor $Q$ is

$$
\frac{\Delta Q}{Q_{m}} \%=\frac{Q_{c}-Q_{m}}{Q_{m}} \times 100
$$

where $Q_{c}$ is the calculated quality factor and $Q_{m}$ is the measured quality factor. From (9), the calculated quality factor is

$$
\begin{aligned}
Q_{c}= & \frac{\omega L\left(1-\omega L C-\frac{C}{L} R_{\mathrm{ac}-c}^{2}\right)}{R_{\mathrm{ac}-c}} \\
= & \frac{\omega L\left[1-\omega L C-\frac{C}{L}\left(R_{\mathrm{ac}-c}+\Delta R_{\mathrm{ac}}\right)^{2}\right]}{R_{\mathrm{ac}-m}+\Delta R_{\mathrm{ac}}} \\
= & \frac{\omega L\left(1-\omega L C-\frac{C}{L} R_{\mathrm{ac}-m}^{2}\right)-\omega C\left(2 R_{\mathrm{ac}-m} \Delta R_{\mathrm{ac}}+\Delta R_{\mathrm{ac}}^{2}\right)}{R_{\mathrm{ac}-m}+\Delta R_{\mathrm{ac}}} \\
= & \omega L\left(1-\omega L C-\frac{C}{L} R_{\mathrm{ac}-m}^{2}\right) \frac{\Delta R_{\mathrm{ac}}}{R_{\mathrm{ac}-m}+\Delta R_{\mathrm{ac}}} \\
& -\frac{\omega C\left(2 R_{\mathrm{ac}-m} \Delta R_{\mathrm{ac}}+\Delta R_{\mathrm{ac}}^{2}\right)}{R_{\mathrm{ac}-m}+\Delta R_{\mathrm{ac}}} \\
= & Q_{m}\left[\frac{R_{\mathrm{ac}-m}}{R_{\mathrm{ac}-m}+\Delta R_{\mathrm{ac}}}-1\right]+Q_{m} \\
& -\frac{\omega C \Delta R_{\mathrm{ac}}\left(2 R_{\mathrm{ac}-m}+\Delta R_{\mathrm{ac}}\right)}{R_{\mathrm{ac}-m}+\Delta R_{\mathrm{ac}}} .
\end{aligned}
$$

This gives

$$
\begin{aligned}
\Delta Q=Q_{m} & \frac{\Delta R_{\mathrm{ac}}}{R_{\mathrm{ac}-m}+\Delta R_{\mathrm{ac}}} \\
& +\omega C_{m} \frac{\Delta R_{\mathrm{ac}}}{R_{\mathrm{ac}-m}+\Delta R_{\mathrm{ac}}}\left(2 R_{\mathrm{ac}-m}+\Delta R_{\mathrm{ac}}\right) .
\end{aligned}
$$

Using (13), the percentage error for the quality factor is expressed as

$$
\Delta Q \% \equiv \cdot \frac{\Delta Q}{Q_{m}} \times 100 \approx \frac{\Delta R_{\mathrm{ac}}}{R_{\mathrm{ac}-m}} \frac{1}{1+\frac{\Delta R_{\mathrm{ac}}}{R_{\mathrm{ac}-m}}} \times 100
$$

which is approximated as

and

$$
\Delta Q \% \approx \frac{\Delta R_{\mathrm{ac}}}{R_{\mathrm{ac}-m}} \times 100, \quad \text { for } \frac{\Delta R_{\mathrm{ac}}}{R_{\mathrm{ac}-m}} \ll 1
$$

$$
\Delta Q \% \approx 100, \text { for } \frac{\Delta R_{\mathrm{ac}}}{R_{\mathrm{ac}-m}} \gg 1 \text {. }
$$

As an example, (2) can be considered. It allows for an accurate prediction of $R_{\mathrm{ac}}$ of inductor no. 1 and, therefore, also the calculated values of the quality factor are in good agreement with experimental values. However, it gives large errors for $R_{\mathrm{ac}}$ of inductor no. 2 , resulting in a poor accurate calculation of the quality factor.

\section{REFERENCES}

[1] P. L. Dowell, "Effects of eddy currents in transformer windings," Proc. Inst. Elect. Eng., vol. 113, pp. 1387-1394, Aug. 1966.

[2] J. Jongsma, "High-frequency ferrite power transformer and choke design, part 3: Transformer winding design, part 4: Improved method of power choke design," in Philips Electronic Components and Materials Technical Publication, Philips, The Netherlands, 1986.

[3] P. S. Venkatraman, "Winding eddy current losses in switch mode power transformers due to rectangular wave currents," in Proc. Powercon 11, sec. A-1. Ventura, CA, 1984, pp. 1-11. 
[4] B. Carsten, "High frequency conductor losses in switch mode magnetics," in Proc. High-Frequency Power Conversion Conf., May 1986, pp. $155-176$.

[5] M. P. Perry, "Multiple layer series connected winding design for minimum losses," IEEE Trans. Power App. Syst., vol. PAS-98, pp. 116-123, Jan./Feb. 1979

[6] E. Bennet and S. C. Larson, "Effective resistance of alternating currents of multilayer windings," Trans. Amer. Inst. Elect. Eng., vol. 59, pp. $1010-1017,1940$

[7] J. P. Vandelac and P. D. Ziogas, "A novel approach for minimizing highfrequency transformer copper losses," IEEE Trans. Power Electron., vol. 3 , pp. 266-276, July 1988.

[8] A. M. Urlig, V. A. Niemela, G. R. Skutt, and T. G. Wilson, "Characterizing high-frequency effects in transformer windings-A guide to several significant articles," in IEEE Appl. Power Electronics Conf. Proc., Mar. 1989, pp. 373-385.

[9] J. A. Ferreira, Electromagnetic Modeling of Power Electronic Converters. Boston, MA: Kluwer, 1989.

[10] - "Improved analytical modeling of conductive losses in magnetic components," IEEE Trans. Power Electron., vol. 9, pp. 127-131, Jan. 1994.

[11] M. Bartoli, N. Noferi, A. Reatti, and M. K. Kazimierczuk, "Modeling winding losses in high-frequency power inductors," World Sci. J. Circ., Syst. Comput. (Special Issue on Power Electronics, Part II), vol. 5, pp. 607-626, Dec. 1996. .

[12] M. Bartoli, A. Reatti, and M. K. Kazimierczuk, "Modeling iron-powder inductors at high frequencies," Proc. 1994 IEEE-IAS Annu. Meeting, vol. 2, pp. 1225-1232, Oct. 2-7, 1994

[13] — - "Predicting the high-frequency ferrite-core inductor performance," in Proc. Electrical Manufacturing and Coil Windings Association Meeting, Chicago, IL, Sept. 27-29, 1994, pp. 409-413.

[14] _ , "High-frequency models of ferrite cores inductors," in Proc. IECON'94, Bologna, Italy, Sept. 5-9, 1994, pp. 1670-1675.

[15] S. Ryzko and J. Ebert, "Effect of a screen on a single layer solenoid at high frequencies," Bull. Acad. Polon. Sci., ser. Science and Technique, no. 8,1965 .
Alberto Reatti received the degree in electronics engineering from the University of Florence, Italy, in 1988, and the Ph.D. degree from the University of Florence in 1993.

In 1992 he was an Associate Researcher with the Department of Electrical Engineering, Wright State University, Dayton, OH. In 1994, he was Visiting Professor with the Department of Electronics at the University of Siena. He has also been Visiting Professor with the Department of Electronics at the University of Florence for several years and, currently, is Associate Professor at the Department of Electronics and Telecommunications at the University of Florence. In 1996/97 he was Chairman of Power Electronic and Power Systems Committee of IEEE ISCAS. His interests are in the high-frequency high-efficiency power converters

Marian K. Kazimierczuk (M'91-SM'91) received the M.S., Ph.D., and D.Sci. degrees in electronics engineering from the Department of Electronics, Technical University of Warsaw, Warsaw, Poland, in 1971, 1978, and 1984, respectively.

He was a Teaching and Research Assistant from 1972 to 1978 and an Assistant Professor from 1978 to 1984 with the Department of Electronics, Institute of Radio Electronics, Technical University of Warsaw. In 1984, he was a Project Engineer for Design Automation, Inc., Lexington, MA. In 1984-85, he was a Visiting Professor with the Department of Electrical Engineering, Virginia Polytechnic Institute and State University, VA. Since 1985, he has been with the Department of Electrical Engineering, Wright State University, Dayton, OH, where he is currently a Professor. His research interests are in resonant and PWM dc/dc power converters, dc/ac inverters, high-frequency rectifiers, electronic ballasts, magnetics, power semiconductor devices, and high-frequency high-efficiency power tuned amplifiers. He is the coauthor of the book Resonant Power Converters (New York: Wiley, 1995). He has published over 200 technical papers, 70 of which have appeared in IEEE transactions and journals.

Dr. Kazimierczuk received the IEEE Harrell V. Noble Award for his contributions to the fields of aerospace, industrial, and power electronics in 1991. $\mathrm{He}$ is also a recipient of the 1991 Presidential Award for Faculty Excellence in Research, the 1993 College Teaching Award, the 1995 Presidential Award for Outstanding Faculty Member, and the Brage Golding Distinguished Professor of Research Award from Wright State University. He was an Associate Editor of the IEEE TRANSACTIONS ON CIRCUITS AND SYSTEMS, PART I and served as an Associate Editor for the JOURNAL OF CIRCUITS, SYSTEMS, AND COMPUTERS. He was a member of the Superconductivity Committee of the IEEE Power Electronics Society. He is a chair of the CAS Technical Committee of Power Systems and Power Electronic Circuits. 